\title{
OSCILLATION AND COMPARISON FOR SECOND ORDER DIFFERENTIAL EQUATIONS
}

\section{KEITH SCHRADER}

ABSTRACT. Consider the equations

$$
x^{\prime \prime}=f\left(t, x, x^{\prime}\right)
$$

and

$$
x^{\prime \prime}=g\left(t, x, x^{\prime}\right)
$$

where $f, g:[a,+\infty) \times R^{2} \rightarrow R$ are continuous. Assume that solutions of initial value problems for (1) and for (2) are unique and extend to $[a,+\infty)$. Let $f(t, 0,0)=0=g(t, 0,0)$ for $t \in[a,+\infty)$ and $f\left(t, x, x^{\prime}\right) / x \leq g\left(t, x, x^{\prime}\right) / x$ for $|x|>0$ and $\left(t, x, x^{\prime}\right)$ in the domain of $f$ and $g$. Under these hypotheses it can be shown that if every solution of (2) has a zero on an interval $I \mathrm{C}$ $[a,+\infty)$ then it follows that every solution of (1) has a zero on $I$. In particular this shows that under the se hypotheses $(2)$ is oscillatory (every solution has a zero on $[a+n,+\infty)$ for each positive integer $n$ ) implies (1) is oscillatory.

1. Introduction. We are concerned with the second order ordinary differential equations

$$
x^{\prime \prime}=f\left(t, x, x^{\prime}\right)
$$

and

$$
x^{\prime \prime}=g\left(t, x, x^{\prime}\right)
$$

where we assume that $f, g:[a,+\infty) \times R^{2} \rightarrow R$ are continuous and that solutions of initial value problems for (1) and for (2) are unique and extend to $[a,+\infty)$. We say that an equation such as $(1)$ is oscillatory on $[a,+\infty)$ if every solution of (1) has zeros for arbitrarily large values of $t$.

We will show in this paper that if $f(t, 0,0)=0=g(t, 0,0)$ for $t \in[a,+\infty)$ and if $f\left(t, x, x^{\prime}\right) / x \leq g\left(t, x, x^{\prime}\right) / x$ for $|x|>0$ and $\left(t, x, x^{\prime}\right)$ in the domain of $f$ and $g$ then (2) is oscillatory implies (1) is oscillatory. In addition, under the same hypotheses, we will show that if every solution of (2) has a zero on

Received by the editors May 10, 1974.

AMS (MOS) subject classifications (1970). Primary 34C10, 34C15; Secondary $34 \mathrm{~B} 15$.

Key words and phrases. Oscillation, comparison, second order, nonlinear. 
an interval $I \subset[a,+\infty)$ then every solution of (1) has a zero on the interval I. This result actually implies the preceding one since (2) oscillatory implies every solution of (2) has a zero on $[a+n,+\infty)$ for each $n=0,1,2, \cdots$ which implies that every solution of (1) has a zero on $[a+n,+\infty)$ for each $n=0,1, \cdots$ which implies (1) is oscillatory.

For a comprehensive survey on oscillation for second order equations we refer the reader to the paper by Wong [4]. Other comparison theorems for oscillatory equations can be found in Utz [2, Theorem 1, p. 56], Waltman [3, Theorem 3, p. 52] and Wong [4, Theorem 5, p. 219] among other places.

2. Comparison theorems. The first result we give is a comparison theorem concerning oscillation which generalizes the result in Wong's survey paper [4, Theorem 5, p. 219].

Theorem 2.1. Let $f, g:[a,+\infty) \times R^{2} \rightarrow R$ be continuous and let solutions of initial value problems for (1) and for (2) be unique and extend to $[a,+\infty)$. If $f(t, 0,0)=0=g(t, 0,0)$ for $t \in[a,+\infty)$ and if $f\left(t, x, x^{\prime}\right) / x \leq$ $g\left(t, x, x^{\prime}\right) / x$ for $|x|>0$ and $\left(t, x, x^{\prime}\right)$ in the domain of $f$ and $g$ then (2) is oscillatory implies (1) is oscillatory.

Proof. If (1) is not oscillatory then there exists a solution $y$ of (1) and a subinterval $[b,+\infty) \subset[a,+\infty)$ such that $y(t)$ is not equal to zero on $[b,+\infty)$ We assume that $y(t)>0$ for $t \in[b,+\infty)$ since the proof of the other case is similar. It follows from [1, Theorem 4.1] using $\psi=y$ and $\phi=0$ that there is a solution $z$ of (2) that satisfies $z(b)=y(b)$ and $0 \leq z(t) \leq y(t)$ for $t \epsilon$ $[b,+\infty)$. This contradicts the fact that (2) is oscillatory since by the uniqueness of solutions to initial value problems and the fact that $z(b)>0$ we must have $0<z(t)$ for $t \geq b$.

We next observe that Theorem 3.1 of [1] is still correct if we only require that there exist functions $\phi, \psi$ in $C[a, b]$ which are piecen (乙) wice continuously differentiable lower and upper solutions respectively. we will state that result next and indicate the modifications needed in the hypotheses and in the proof. Since we do not need the full generality that is given in [ 1 , Theorem 3.1] we simplify the result somewhat and only state the sufficiency part corresponding to part (i). Notice that our assumption about solutions extending to $[a,+\infty)$ implies that condition (i) of $[1$, Theorem 3.1$]$ will be satisfied.

Theorem 2.2. Let $g:[a,+\infty) \times R^{2} \rightarrow R$ be continuous and let solutions of initial value problems for (2) be unique and extend to $[a,+\infty)$. Let $[c, d]$ $\subset[a,+\infty)$ and let $\alpha, \beta$ be given numbers. Assume that there exist functions 
$\phi, \psi \in C[c, d]$ with $\phi(t) \leq \psi(t)$ on $[c, d], \phi(c) \leq \alpha \leq \psi(c)$ and $\phi(d) \leq \beta \leq$ $\psi(d)$. Assume there exist numbers $c=t_{0}<t_{1}<\cdots<t_{s}=d$ and $c=\tau_{0}<\tau_{1}$ $<\cdots<\tau_{r}=d$ such that $\phi \in C^{2}\left[t_{i-1}, t_{i}\right]$ for $i=1, \cdots, s, \psi \in C^{2}\left[r_{i-1}, \tau_{i}\right]$ for $i=1, \cdots, r$ and such that $\phi$ is a lower solution for (2) on $\left[t_{i-1}, t_{i}\right]$ for $i=$ $1, \cdots, s$ and $\psi$ is an upper solution for (2) on $\left[\tau_{i-1}, \tau_{i}\right]$ for $i=1, \cdots, r$. If $\phi^{\prime}\left(t_{i}-\right)<\phi^{\prime}\left(t_{i}+\right)$ for $i=1, \cdots, s-1$ and $\psi^{\prime}\left(\tau_{i^{-}}\right)>\psi^{\prime}\left(\tau_{i^{+}}\right)$for $i=1, \cdots$, $r-1$ then the boundary value problem (2) with boundary conditions

$$
x(c)=\alpha, \quad x(d)=\beta
$$

has a solution $z$ in $C^{2}[c, d]$ and $\phi(t) \leq z(t) \leq \psi(t)$ for $c \leq t \leq d$.

Proof. The proof proceeds exactly as the proof of Theorem 3.1 of [1] except that $N>1$ should be chosen large enough so that $\left|\phi^{\prime}(t)\right| \leq N$ for $t_{i-1} \leq$ $t \leq t_{i}$ and for $i=1, \cdots ; s$ and such that $\left|\psi^{\prime}(t)\right| \leq N$ for $\tau_{i-1} \leq t \leq \tau_{i}$ and for $i=1, \cdots, r$. The argument that the solution $y_{N}$ in the proof of Theorem 3.1 of [1] satisfies $\phi(t) \leq y_{N}(t) \leq \psi(t)$ is essentially an argument that the second derivative of $y_{N}-\psi$ or $\phi-y_{N}$ would have the wrong sign at a positive maximum otherwise. This argument is still valid because any positive maximum of $y_{N}-\psi$, for example, could not occur at any $\tau_{i}$ for $i=1, \cdots, r-1$ since $\left(y_{N}-\psi\right)^{\prime}\left(\tau_{i}\right) \leq 0$ and $\left(y_{N}-\psi\right)^{\prime}\left(\tau_{i}\right) \geq 0$ at a positive maximum at $\tau_{i}$ would imply $\psi^{\prime}\left(\tau_{i}-\right) \leq y_{N}^{\prime}\left(\tau_{i}\right) \leq \psi^{\prime}\left(\tau_{i}+\right)$ which would contradict the condition $\psi^{\prime}\left(\tau_{i}{ }^{+}\right)$ $\left\langle\psi^{\prime}\left(r_{i}-\right)\right.$. The rest of the proof is the same as the proof of Theorem 3.1 of [1].

The result, Theorem 4.1 of $[1]$ on $[c,+\infty) \subset[a,+\infty)$, may also be stated for piecewise twice continuously differentiable functions provided the inequalities on the one sided derivatives of $\phi$ and $\psi$ at the points $t_{i}$ and $\tau_{i}$, respectively, are required to hold as in Theorem 2.2. A similar result holds on a finite half open interval of the form $[c, d) \subset[a,+\infty)$ or $(c, d] \subset[a,+\infty)$ where one boundary condition is specified at $c$ or $d$, respectively. When we wish to refer to any of these results we will just refer to the appropriate analogue of Theorem 2.2 for the interval in question.

The next result is our main theorem and, as pointed out in $\$ 1$, this result implies Theorem 2.1.

Theorem 2.3. Let $f, g$ satisfy the bypotheses of Theorem 2.1 and let $I C$ $[a,+\infty)$ be an interval. If every solution of (2) has a zero on $I$, then every solution of (1) has a zero on $I$.

Proof. Let $y$ be a solution of (1) having no zero on the interval $I$. We will assume that $y(t)>0$ for $t \in I$ since the proof of the other case is similar. If $i=[z,+\infty)$ the proof follows as in the proof of Theorem 2.1. 
If $I=[c, d]$ then it follows from Theorem 3.1 of [1] that there is a solution $z$ of (2) which satisfies $z(c)=y(c), z(d)=y(d)$ and $0 \leq z(t) \leq y(t)$ for $t \in[c, d]$. Moreover, since $z(c)>0$ and $z(d)>0$ it follows from the uniqueness of solutions to initial value problems that $z(t)>0$ for $t \in[c, d]$. This contradicts the fact that every solution of (2) has a zero on $I$.

If $l=[c, d)$ then the appropriate analogue of Theorem 2.2 using $\phi=0$ and $\psi=y$ gives a solution $z$ of (2) which satisfies $z(c)=y(c)$ and $0 \leq z(t)$ $\leq y(t)$ for $t \in[c, d)$. Uniqueness of solutions to initial value problems implies $z(t)>0$ for $t \in[c, d)$. This contradicts the fact that every solution of (2) has a zero on $I$.

If $I=(c, d]$ the proof is similar to the case just considered.

If $I=(c, d)$ then we may assume that $y(c)=0$ for otherwise this case may be treated like the case for $I=[c, d)$. Similarly we assume $y(d)=0$ for otherwise the treatment as for the case $I=(c, d]$ works. Note that $y^{\prime}(c) \neq 0$ by uniqueness of solutions to initial value problems for (2). For a positive integer $n$ let $y_{n}$ be a solution of the initial value problem for (2) with initial conditions $y_{n}(c)=0, y_{n}^{\prime}(c)=1 / n$ where $0<1 / n<y^{\prime}(c)$. Let $t_{1}(n)$ be the smallest value of $t, c<t<d$, for which $y_{n}(t)=0$. If for some $n, y_{n}(t)<y(t)$ for $t \in\left[c, t_{1}(n)\right]$ then by Theorem 2.2 using $\psi=y$ and $\phi=y_{n}$ for $c \leq t \leq$ $t_{1}(n)$ and $\phi=0$ for $t_{1}(n)<t \leq d$ it follows that there is a solution $z$ of (2) which satisfies $z(c)=0, z(d)=0$ and $\phi(t) \leq z(t) \leq y(t)$ for $t \in[c, d]$. It follows from the uniqueness of solutions to initial value problems that $z(t)>0$ for $t \in(c, d)$. This contradicts the fact that every solution of (2) has a zero on $(c, d)$. We conclude that for each $n$ there is a $\tau(n)<t_{1}(n)$ such that $y_{n}(\tau(n))=y(\tau(n))$. Since $y_{n}$ converges uniformly to zero on $[c, d]$ it follows that we may choose a subsequence of $\{n\}$, again denoted by $\{n\}$, such that $\tau(n) \rightarrow c$ as $n \rightarrow+\infty$ or else such that $\tau(n) \rightarrow d$ as $n \rightarrow+\infty$. We consider only the second case since the proof for the first case is similar. Now by the mean value theorem we have

$$
y_{n}^{\prime}\left(\gamma_{n}\right)=\frac{y_{n}(\tau(n))-y_{n}\left(t_{1}(n)\right)}{\tau(n)-t_{1}(n)}=\frac{y(\tau(n))-y(d)}{\tau(n)-t_{1}(n)} \leq \frac{y(\tau(n))-y(d)}{\tau(n)-d}=y^{\prime}\left(\eta_{n}\right)
$$

where $\tau(n)<\gamma_{n}<t_{1}(n)$ and $\tau(n)<\eta_{n}<d$. It follows then that

$$
\limsup _{n \rightarrow+\infty} y_{n}^{\prime}\left(\gamma_{n}\right) \leq y^{\prime}(d)<0
$$

but this contradicts the fact that $y_{n}^{\prime}\left(\gamma_{n}\right)$ must converge to zero. We conclude that every solution of (1) has a zero on $(c, d)$. 
If $I=(c,+\infty)$ we may assume that $y(c)=0$ for otherwise this case may be treated like the case when $I=[c,+\infty)$. For $n$ an integer small enough so that $0<1 / n<y(c+1)$ apply Theorem 4.1 of [1] to the interval $[c+1,+\infty)$ using $\phi=0$ and $\psi=y$ to get a solution $y_{n}$ of (2) which satisfies the boundary conditions $y_{n}(c+1)=1 / n$ and $0 \leq y_{n}(t) \leq y(t)$ for $t \in[c+1,+\infty)$. Now apply the mean value theorem on $[c+1, c+2]$ to get

$$
y_{n}^{\prime}\left(\lambda_{n}\right)=\frac{y_{n}(c+2)-y_{n}(c+1)}{(c+2)-(c+1)}=y_{n}(c+2)-y_{n}(c+1)
$$

for some $\lambda_{n}$ with $c+1<\lambda_{n}<c+2$ so that $\left|y_{n}^{\prime}\left(\lambda_{n}\right)\right|$ is bounded and we may choose a subsequence $\{n(k)\}$ so that $\left\{\lambda_{n(k)}\right\},\left\{y_{n(k)}\left(\lambda_{n(k)}\right)\right\}$ and $\left\{y_{n(k)}^{\prime}\left(\lambda_{n(k)}\right)\right\}$ converge. We will denote the sequence $\{n(k)\}$ by $\{n\}$ again to simplify notation. Thus $\left\{y_{n}\right\}$ converges to a solution $z$ of (2) with $z(c+1)=0$ and $0 \leq$ $z(t) \leq y(t)$ for $t \in[c+1,+\infty)$. If $z$ is not identically zero on $[c+1,+\infty)$ then apply the appropriate analogue of Theorem 2.2 to the interval $[c,+\infty)$ using $\psi=y$ and $\phi=0$ for $c \leq t \leq c+1$ and $\phi=z(t)$ for $c+1<t<+\infty$. This yields a solution $w$ of (2) which satisfies $w(c)=0, \phi(t) \leq w(t) \leq y(t)$ for $t \in[c,+\infty)$. It follows by uniqueness of solutions to initial value problems that $w(t)>0$ for $t \in(c,+\infty)$ which contradicts the fact that all solutions of (2) have a zero on $(c,+\infty)$. We conclude that $z(t)=0$ for $t \in[c+1,+\infty)$ and hence by uniqueness of solutions to initial value problems, $z(t) \equiv 0$ for $t \epsilon$ $[c,+\infty)$. Let $t_{1}(n)$ be the largest value of $t, c<t<c+1$, for which $y_{n}(t)=$ 0 . If for some $n, y_{n}(t)<y(t)$ for $t \in\left[t_{1}(n), c+1\right]$ then by the appropriate analogue of Theorem 2.2 applied to the interval $[c,+\infty)$ using $\psi=y$ and $\phi=0$ for $c \leq t \leq t_{1}(n)$ and $\phi=y_{n}(t)$ for $t_{1}(n)<t<+\infty$ there would be a solution $u$ of (2) satisfying $u(c)=0$ and $\phi(t) \leq u(t) \leq y(t)$ for $t \in[c,+\infty)$. By uniqueness of solutions to initial value problems it would follow that $u(t)>0$ for $t \epsilon$ $(c,+\infty)$ which would be a contradiction. We conclude that for each $n$ there is a $\tau(n)>t_{1}(n)$ such that $y_{n}(\tau(n))=y(\tau(n))$. Now by the mean value theorem we have

$$
y_{n}^{\prime}\left(\gamma_{n}\right)=\frac{y_{n}(\tau(n))-y_{n}\left(t_{1}(n)\right)}{\tau(n)-t_{1}(n)}=\frac{y(\tau(n))-y(c)}{\tau(n)-t_{1}(n)} \geq \frac{y(\tau(n))-y(c)}{\tau(n)-c}=y^{\prime}\left(\eta_{n}\right)
$$

where $t_{1}(n)<\gamma_{n}<\tau(n)$ and $c<\eta_{n}<\tau(n)$. Since $y_{n}$ converges uniformly to zero on $[c, c+1]$ it follows that $\tau(n) \rightarrow c$ as $n \rightarrow+\infty$. It then follows that lim inf ${ }_{n \rightarrow+\infty} y_{n}^{\prime}\left(\gamma_{n}\right) \geq y^{\prime}(c)>0$ but this contradicts the fact that $y_{n}^{\prime}\left(\gamma_{n}\right)$ must converge to zero. We conclude that every solution of (1) has a zero on $I=$ $(c,+\infty)$. This completes the proof. 


\section{REFERENCES}

1. K. W. Schrader, Existence theorems for second order boundary value problems, J. Differential Equations 5 (1969), 572-584. MR 39 \#532.

2. W. R. Utz, Properties of solutions of $u^{\prime \prime}+g(t) u^{2 n-1}=0$, Monatsh. Math. 66 (1962), 55-60. MR 25 \#2275.

3. P. Waltman, Some properties of solutions of $u^{\prime \prime}+a(t) f(u)=0$, Monatsh. Math. 67 (1963), 50-54. MR $26 \# 5214$.

4. J. S. W. Wong, On second order nonlinear oscillation, Funkcial Ekvac. 11 (1968), 207-234 (1969). MR $39 \# 7221$.

DEPARTMENT OF MATHEMATICS, UNIVERSITY OF MISSOURI, COLUMBIA, MISSOURI 65210 